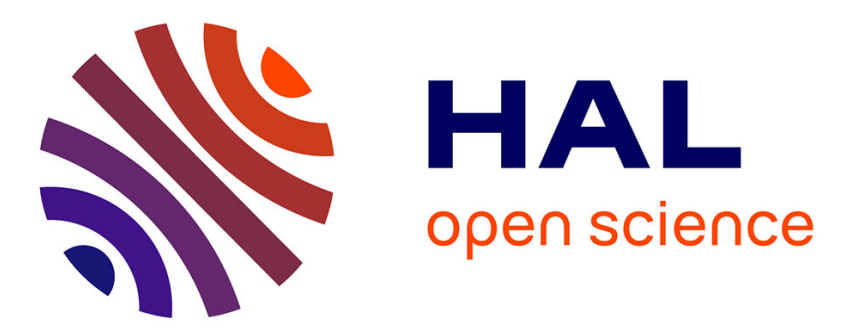

\title{
Synchronized diffusive-wave spectroscopy: Principle and application to sound propagation in aqueous foams
}

Jérôme Crassous, Patrick Chasle, Juliette Pierre, Arnaud Saint-Jalmes, Benjamin Dollet

\section{- To cite this version:}

Jérôme Crassous, Patrick Chasle, Juliette Pierre, Arnaud Saint-Jalmes, Benjamin Dollet. Synchronized diffusive-wave spectroscopy: Principle and application to sound propagation in aqueous foams. Physical Review E , 2016, 93 (3), pp.032611. 10.1103/PhysRevE.93.032611 • hal-01313968

HAL Id: hal-01313968

https://hal-univ-rennes1.archives-ouvertes.fr/hal-01313968

Submitted on 14 Jun 2016

HAL is a multi-disciplinary open access archive for the deposit and dissemination of scientific research documents, whether they are published or not. The documents may come from teaching and research institutions in France or abroad, or from public or private research centers.
L'archive ouverte pluridisciplinaire HAL, est destinée au dépôt et à la diffusion de documents scientifiques de niveau recherche, publiés ou non, émanant des établissements d'enseignement et de recherche français ou étrangers, des laboratoires publics ou privés. 


\title{
Synchronized Diffusive Wave Spectroscopy: Principle and Application to Sound Propagation in Aqueous Foams
}

\author{
Jérôme Crassous, ${ }^{*}$ Patrick Chasle, Juliette Pierre, Arnaud Saint-Jalmes, and Benjamin Dollet \\ Institut de Physique de Rennes, UMR CNRS 6251, Université de Rennes 1, \\ Campus de Beaulieu, F-35042 RENNES Cedex, France
}

(Dated: January 18, 2016)

\begin{abstract}
We present an experimental method to measure oscillatory strains in turbid material. The material is illuminated with a laser, and the speckle patterns are recorded. The analysis of the deformations of the optical pathlength shows that the speckle patterns are modulated at the strain frequency. By recording those patterns synchronously with the strain source, we are able to measure the amplitude and the phase of the strain. This method is tested on the specific case of an aqueous foam where an acoustic wave propagates. The effects of material internal dynamics and of heterogeneous deformations are also discussed.
\end{abstract}

PACS numbers: 42.25.Dd Wave propagation in random media, 78.35.+c Brillouin and Rayleigh scattering; other light scattering, 62.20.F- Deformation and plasticity 83.80.Iz Emulsions and foams

\section{INTRODUCTION}

Soft materials are commonly used in the food, cosmetics and pharmaceutical industries; this is in part due to the wide range of texture and rheology which can be encountered when dealing with gels, pastes, concentrated colloidal solutions, foams or emulsions. Together with these mechanical aspects, the ability to encapsulate and deliver chemicals provides some increased interest in such materials. As well, the non-trivial and often dispersive nature of wave propagation in such materials is also another specificity which can be optimized to get original thermal or acoustical macroscopic properties. The drawbacks of such a high potentiality in applications are the structural and dynamical complexity of such soft materials; they are disordered, often opaque, and generally driven in a out-of-equilibrium state as they are formed. This results in time evolution (aging) and complex intrinsic dynamical mechanisms, with possible intermittency and heterogeneity in space and time. Understanding these evolutions inside the materials remains a generic and important issue. It is for instance important to determine the microscopic origins of the intrinsic rearrangements, and how they can be coupled and superimposed onto rearrangements induced by external forcing. As well, transport properties and wave propagation in such disordered and amorphous materials need to be investigated, both on fundamental and practical standpoints. Numerous techniques to scan these materials have been developed, especially light scattering methods, taking advantage of being non-intrusive. When dealing with turbid media, diffusive wave spectroscopy (DWS) is well suited, as it can monitor the internal dynamics in highly diffusive samples $[1,2]$. This technique has been improved in different ways to better resolve the space and time fluctuations, to reduce the measurement timescales, and to

* jerome.crassous@univ-rennes1.fr get more information on the homogeneity of the dynamics [3-9]. Despite these improvements, the optimization of existing light scattering methods or the design of new ones remains an active axis of research.

As a typical example of soft, opaque and aging materials, aqueous foams - dispersions of gas into a liquid have been used as model systems to test and demonstrate the efficiency of DWS $[10,11]$. With time, DWS actually became a major tool for investigating foams and has provided important insights on foam properties at the scale of the bubbles, especially when it was coupled to macroscopic rheology $[12,13]$. However, and despite their wide use in industries and active academic studies [14, 15], various issues on aqueous foams remain open. These pending problems mostly concern (i) the time destabilization of a foam and how this can be controlled by the physicochemical parameters, and (ii) the rheology of foams and its specificities when compared to other soft glassy materials. In that respect, new progress requires techniques to follow how the bubble diameter $D$ and the foam liquid fraction $\phi_{l}$ evolve in time; in fact, as a consequence of drainage and coarsening, these two crucial quantities can hardly be kept constant, as the bubble diameter increases with time and the initial liquid content of the foam decreases with time. Optical and electrical methods can be used to monitor these aging effects, but the whole evolution of $D$ and $\phi_{l}$ can still not be simply inferred with these techniques. More recently, the acoustic properties of aqueous foams have also been widely investigated, with the aim to design original methods to probe foam properties [16-21]. The propagation of sound in foams turns out to be quite complex, with non-trivial effects, like the occurrence of negative density [20]. Though promising, the experimental approach based on transducers add also some experimental limitations due to the mismatch of acoustic impedance between the transducer and the foam; hence, the injected acoustic wave depends on the foam properties itself. As a consequence, measuring all the sound features into a foam remains tricky. As an alternative, the direct measurement of the acoustic de- 
formation (amplitude and phase) at any given location is another approach to extract the sound velocity and its attenuation. Along this direction, we previously showed that the acoustic deformation can be detected by DWS [22]. This was a first attempt to use light scattering techniques to investigate the sound propagation into a foam.

The method described in [22] allows only to obtain the amplitude of the acoustic waves. The phase of the wave may be obtained by synchronizing the acquisition of scattered light with the acoustic signal. This has been done by Wintzenrieth et al [23]. These authors used a method based on the visibility of the speckle pattern [9]. The variations of the phase difference between the beginning of time integration and the harmonic excitation produces a variation of the visibility. The relative phase between the excitation and material deformation may then be obtained.

Following those previous works, we propose here another DWS experimental scheme where the deformation amplitude and phase can be inferred from synchronized measurements and the combination of four partial correlation functions. The system chosen to make this proof of concept consists in an aqueous foam, where the shear arises from an acoustic forcing. This method is tested on the acoustics propagation into a liquid foam and compared to previous measurements.

In this article, we first describe this DWS scheme, named "Synchronized DWS", and present the mathematical formalism - valid for any diffusive system under a sinusoidal shear deformation - showing that the shear amplitude and phase of this deformation can be derived by measuring four different correlation functions, monitored synchronously with the deformation. Then, we explain how this can be tested by doing experiments on foams under an acoustic forcing. Various results are presented, either at a given location for different imposed amplitude and phase, or while scanning the foam as a function of the distance from the source. Further experimental tests, discussions and comparisons are given in the last section.

\section{SYNCHRONIZED DIFFUSING WAVE SPECTROSCOPY}

\section{A. Principle of the method}

Diffusing wave spectroscopy is a experimental tool which has been successfully used for concentrated colloidal suspensions, emulsions, foams or granular materials. The principle of DWS is to record multiply scattered coherent light, and to monitor the time evolution of this scattered light, in terms of correlation functions. Shortly speaking, the correlation of the scattered intensity arises from interference between many photon paths. If a system is at rest, the interference pattern is frozen, and the scattered intensity does not show temporal evolution. If scatterers move inside the sample, interference (a)

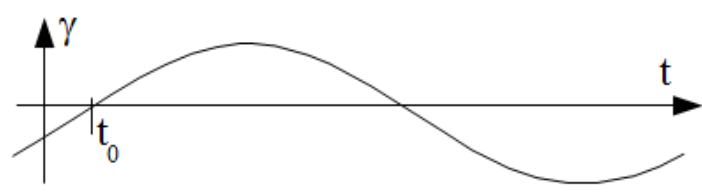

(b)

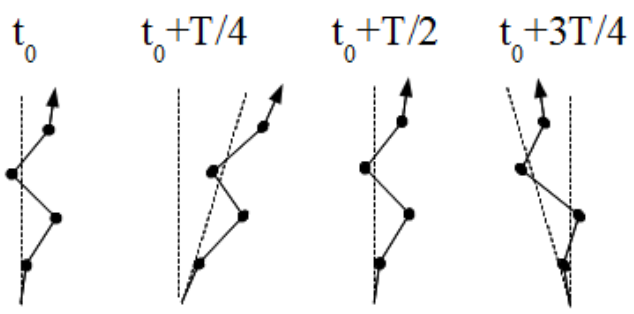

(c)

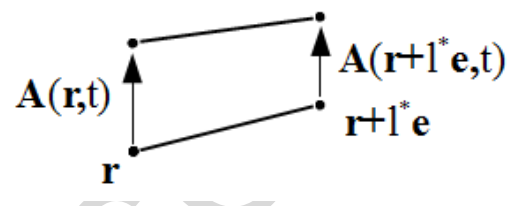

FIG. 1. (a) A sinusoidal shear of displacement $\gamma$. Time $t_{0}$ is such that $\gamma\left(t_{0}\right)=0$. (b) Snapshots of a portion of optical path (full line) on some scatterers (black circle). The zone is periodically sheared and the path periodically deformed. (c) Variation of a segment joining two points $\mathbf{r}$ and $\mathbf{r}+l^{*} \mathbf{e}$. $\mathbf{A}$ is the displacement field of the scatterers.

pattern fluctuates, and the intensity correlation function decreases with time. We consider here the case of a physical system of scatterers which is periodically sheared at frequency $T$. We plot in Fig. 1a a sinusoidal shear deformation. Fig. 1b shows an optical path joining some scatterers. Since they are displaced into the sheared zone, the path is also periodically deformed. Let $t_{0}$ be a time at which the strain vanishes. The paths at times $t_{0}+T / 4$ and $t_{0}+3 T / 4$ are symmetrically sheared. This shear induce a phase shift along this ray, thus a loss of correlation of the scattered light between these two times. The amplitude of the shear may then be related to this loss of correlation. Because $\gamma\left(t_{0}\right)=\gamma\left(t_{0}+T / 2\right)$, the paths at times $t_{0}$ and $t_{0}+T / 2$ are identical. The system appears frozen, and the intensity of the scattered line is perfectly correlated. Hence, the time $t_{0}$ may be deduced from the maximum of correlation of speckle patterns acquired at time $t$ and $t+T / 2$. The phase difference between the shear at a given point of the material and the source of the shear may then be estimated. Therefore, in principle, by a synchronized monitoring at times $t, t+T / 4, t+T / 2$ and $t+3 T / 4$, one can derive the amplitude and the phase of the deformation. The derivation of the relevant mathematical combinations allowing us to get these quantities from the partial correlations is given below. The principle of parallel lock-in detection that we use is not new and has been used in detection of ultrasonic modulation such as described in [24, 25], but to our knowledge never used with DWS. 


\section{B. Link between the intensity correlation function and material deformation}

In the following, we note $l^{*}$ the transport mean free path into the material, $f=1 / T$ the frequency of the strain modulation and $\omega=2 \pi f$. The length of a photon path into the sample is noted $s$. We consider here a parallel detection of the scattered light. For this a camera is used as sensor, and the intensity of many pixels are measured simultaneously. We note $I(t)$ the array of scattered intensity acquired at a time $t$. We compute the normalized correlation function of the scattered intensity:

$$
g_{I}(t, t+\tau)=\frac{\langle I(t) I(t+\tau)\rangle-\langle I\rangle^{2}}{\left\langle I^{2}\right\rangle-\langle I\rangle^{2}},
$$

where $\langle\cdot\rangle$ is here an average on the pixels of the camera. The electronic noise of the camera is removed following a procedure explained in [26]. The normalization of the correlation function ensured that $g_{I}(t, t)=1$ whatever the contrast of the image. From the Siegert relation $g_{I}(t, t+\tau)=\left|g_{E}(t, t+\tau)\right|^{2}$ we obtain the normalized correlation function of the scattered electric field $\mathbf{E}: g_{E}(t, t+\tau)=\left(1 / I_{0}\right)\left\langle\mathbf{E}^{*}(t) \cdot \mathbf{E}(t+\tau)\right\rangle$. The normalization constant $I_{0}$ is the mean scattered intensity.

The autocorrelation function of the scattered electric field may be decomposed as a sum on paths of length $s[1,2]$ :

$$
g_{E}(t, t+\tau)=\int_{s} P(s)\left\langle\exp \left(\Delta \phi_{s}(t, t+\tau)\right)\right\rangle d s .
$$

In (2), $P(s)$ is the normalized path length distribution with $\int_{s} P(s) d s=1$. The complex exponential function is averaged on all paths of length $s$, and $\Delta \phi_{s}(t, t+\tau)$ is the phase shift for a path of length $s$. We drop in the following the two times $t$ and $t+\tau$ in equations. Such paths may be decomposed in $s / l^{*}$ independent segments of length $l^{*}$. It then follows that $\Delta \phi_{s}=\sum_{j=1}^{s / l^{*}} \Delta \phi_{j}$, with $\Delta \phi_{j}$ the phase variation of the segment number $j$ between times $t$ and $t+\tau$. Since $\Delta \phi_{s}$ is the sum of many independent variables, it is a Gaussian random variable, and then:

$$
\begin{aligned}
\left\langle\exp \left(\Delta \phi_{s}\right)\right\rangle & =\exp \left[-\frac{1}{2}\left\langle\Delta \phi_{s}^{2}\right\rangle\right] \\
& =\exp \left[-\frac{1}{2} \sum_{j=1}^{s / l^{*}}\left\langle\Delta \phi_{j}^{2}\right\rangle\right] .
\end{aligned}
$$

In $(3),\langle\cdot\rangle$ is an average on all orientations of the segments, and we also assume that $\left\langle\Delta \phi_{j}\right\rangle=0$, i.e. there is no dilatation of compression of the medium. If the deformation is homogeneous, $\left\langle\Delta \phi_{j}^{2}\right\rangle$ does not depend on the number of the segment, and $\sum_{j=1}^{s / l^{*}}\left\langle\Delta \phi_{j}^{2}\right\rangle=s\left\langle\Delta \phi^{2}\right\rangle / l^{*}$. For small enough phase shifts, $\exp \left[s\left\langle\Delta \phi^{2}\right\rangle / 2 l^{*}\right] \simeq 1-$ $s\left\langle\Delta \phi^{2}\right\rangle / 2 l^{*}$ and (2) becomes: $g_{E}(t, t+\tau) \simeq \int_{s} P(s)[1-$ $\left.s\left\langle\Delta \phi^{2}\right\rangle / 2 l^{*}\right] d s$. After integration we obtain:

$$
g_{E}(t, t+\tau) \simeq 1-A\left\langle\Delta \phi^{2}(t, t+\tau)\right\rangle,
$$

with $A$ a constant depending on $P(s)$. Following the approach detailed in numerous studies [27? -29] we now relate $\left\langle\Delta \phi^{2}\right\rangle$ to the deformation of the material. We consider a segment joining the points $\mathbf{r}$ and $\mathbf{r}+l^{*} \mathbf{e}$ with e the unit vector joining the two points as shown on Fig. 1c. The displacements at time $t$ are $\mathbf{A}(\mathbf{r}, t)$ and $\mathbf{A}\left(\mathbf{r}+l^{*} \mathbf{e}, t\right)$, and the new distance between the two points is $\simeq l^{*}(1+[(\mathbf{e} \cdot \nabla) \mathbf{A}(\mathbf{r}, t)] \cdot \mathbf{e})$. The variation of the phase between the two times $t$ and $t+\tau$ is then $k$ times the difference of length:

$$
\Delta \phi=k l^{*}[(\mathbf{e} \cdot \nabla)(\mathbf{A}(\mathbf{r}, t+\tau)-\mathbf{A}(\mathbf{r}, t))] \cdot \mathbf{e} .
$$

For a harmonic displacement with a polarization along the unit vector $\mathbf{a}$, we can write $\mathbf{A}(\mathbf{r}, t)=[\alpha(\mathbf{r}) \cos (\omega t)+$ $\beta(\mathbf{r}) \sin (\omega t)] \mathbf{a}$. The phase variation is then:

$$
\begin{aligned}
\Delta \phi & \simeq 2 k l^{*}(\mathbf{e} \cdot \mathbf{a}) \sin (\omega \tau / 2) \\
{[} & -(\mathbf{e} \cdot \nabla \alpha(\mathbf{r})) \sin (\omega t+\omega \tau / 2) \\
& +(\mathbf{e} \cdot \nabla \beta(\mathbf{r})) \cos (\omega t+\omega \tau / 2)] .
\end{aligned}
$$

For simplicity, we suppose that $\nabla \alpha(\mathbf{r})$ and $\nabla \beta(\mathbf{r})$ are parallel to the same unit vector $\mathbf{n}$. We will justify this assumption for our experiments in Sec. IV C. We may then write:

$$
\begin{aligned}
& \nabla \alpha(\mathbf{r})=\gamma(\mathbf{r}) \cos \left(\Psi_{0}(\mathbf{r})\right) \mathbf{n} \\
& \nabla \beta(\mathbf{r})=-\gamma(\mathbf{r}) \sin \left(\Psi_{0}(\mathbf{r})\right) \mathbf{n},
\end{aligned}
$$

with $\gamma(\mathbf{r})$ the amplitude and $\Psi_{0}(\mathbf{r})$ the phase of the deformation. Performing average on segment orientations:

$$
\begin{aligned}
\left\langle\Delta \phi^{2}\right\rangle & \simeq 4 k^{2} l^{* 2} \eta(\mathbf{a}, \mathbf{n}) \gamma^{2}(\mathbf{r}) \sin ^{2}(\omega \tau / 2) \\
& \times \sin ^{2}\left(\omega t+\omega \tau / 2+\Psi_{0}(\mathbf{r})\right)
\end{aligned}
$$

with $\eta(\mathbf{a}, \mathbf{n})=\left\langle(\mathbf{e} \cdot \mathbf{a})^{2}(\mathbf{e} \cdot \mathbf{n})^{2}\right\rangle_{\mathbf{e}}$. For transverse waves $\eta(\mathbf{a}, \mathbf{n})=2 / 15$. Combining with (7), we finally obtain:

$$
\begin{aligned}
\gamma^{2}(\mathbf{r}) \sin ^{2}(\omega \tau / 2) & \times \sin ^{2}\left(\omega t+\omega \tau / 2+\Psi_{0}(\mathbf{r})\right) \\
& \simeq C \times\left[1-g_{E}(t, t+\tau)\right],
\end{aligned}
$$

with $C$ a constant depending on geometry and of optical constants.

\section{Synchronized acquisition}

The correlation functions are now measured at four different combinations of times $t(\bmod T)$ and $t+\tau(\bmod$ $T)$ :

$$
\begin{aligned}
& g^{(1)}=g_{E}(0, T / 2) \\
& g^{(2)}=g_{E}(T / 4,3 T / 4) \\
& g^{(3)}=g_{E}(0, T / 4) \\
& g^{(4)}=g_{E}(T / 4, T / 2) .
\end{aligned}
$$

With (9), we have: 


$$
\begin{aligned}
C \times\left[1-g^{(1)}\right] & =\gamma^{2}(\mathbf{r}) \sin ^{2}\left[\Psi_{0}(\mathbf{r})+\pi / 2\right] \\
C \times\left[1-g^{(2)}\right] & =\gamma^{2}(\mathbf{r}) \sin ^{2}\left[\Psi_{0}(\mathbf{r})+\pi\right] \\
C \times\left[1-g^{(3)}\right] & =\frac{1}{2} \gamma^{2}(\mathbf{r}) \sin ^{2}\left[\Psi_{0}(\mathbf{r})+\pi / 4\right] \\
C \times\left[1-g^{(4)}\right] & =\frac{1}{2} \gamma^{2}(\mathbf{r}) \sin ^{2}\left[\Psi_{0}(\mathbf{r})+3 \pi / 4\right],
\end{aligned}
$$

and then:

$$
\begin{aligned}
\gamma^{2}(\mathbf{r}) & =C \times\left[2-g^{(1)}-g^{(2)}\right] \\
\cot 2 \Psi_{0}(\mathbf{r}) & =\left[\frac{g^{(1)}-g^{(2)}}{2-g^{(1)}-g^{(2)}}\right] /\left[\frac{g^{(3)}-g^{(4)}}{2-g^{(3)}-g^{(4)}}\right] .
\end{aligned}
$$

The amplitude and the phase of the shear may then be simply obtained with the above combinations of correlation functions, all measured synchronously with the harmonic excitation.

\section{EXPERIMENTS}

\section{A. Experimental Setup}

\section{loudspeaker $\sqsupseteq$}

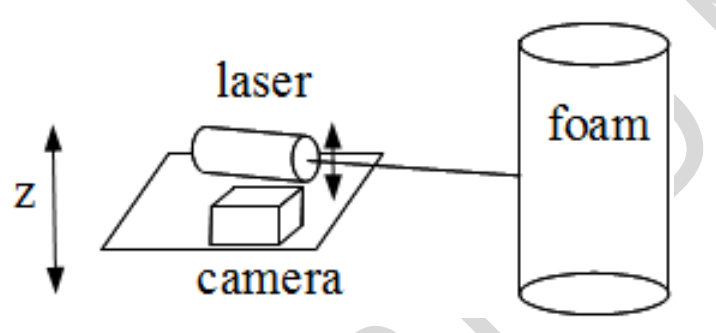

FIG. 2. Schematic drawing of the setup. A cylindrical glass cell is filled with a liquid foam. A loudspeaker generates an acoustic wave. A laser illuminates the foam and the scattered light is detected by a camera. A lens is placed to expand the beam. The camera and the laser may be translated along the $z$ direction.

The preceding formalism is in principle applicable to every scattering medium which is periodically sheared. In order to test this formalism, we performed experiment on aqueous foams, in which we apply a sinusoidal deformation by an external acoustic forcing. In our geometry, the strain occurs because there is a displacement field generated by the acoustic wave into the bulk, and no acoustic displacement at the glass walls [22]. This creates a periodically sheared layer near the lateral glass boundaries. The structure of this sheared layer will be discussed in Sec. IV C.

The experimental setup is drawn on Fig. 2. A cylindrical glass container (diameter $19 \mathrm{~cm}$, height $50 \mathrm{~cm}$ ) is filled with a liquid foam. A loudspeaker is placed above the cylinder, and generates a acoustic wave at a frequency $f$. The acoustic wave emitted by the loudspeaker is partially reflected by the foam surface, and partially transmitted within the foam. The sheared layer is illuminated with the beam of a continuous laser $(633 \mathrm{~nm}$, $15 \mathrm{~mW}$ ). The beam is slightly expanded to a diameter of a few millimeters and illuminates the foam. The scattered light is recorded with a CSMOS camera (PhotonFocus MV1-D1312-160-CL) operating at a frequency $4 f$ : sequences of images at $4 f$ are acquired in a burst mode. The beginning of the acquisition sequence is triggered synchronously to the loudspeaker excitation. A delay between the trigger and the first acquired image may be added in order to imposed a given phase shift $\psi_{0}^{(i m p)}$. All the experiments presented here have been performed at frequency $f=800 \mathrm{~Hz}$ and the exposure time of every image is $11 \mu \mathrm{s}$. The number of pixels of each image is $N_{p}=13120$.

The liquid foam is made using the method described in [30]: it provides large quantities of homogeneous foam, with initial bubble size of the order of 100 microns in diameter. The production rate is $0.1 \mathrm{~L} / \mathrm{s}$, so that our $50-\mathrm{cm}$ high cell is filled in $1 \mathrm{~min}$. The gas used is $\mathrm{C}_{2} \mathrm{~F}_{6}$, to slow down the coarsening [31]. Experiments are performed after waiting times between $30 \mathrm{mn}$ to 5 hours, corresponding to bubble diameter of 200 to 700 microns. For the experimental results shown here, we used sodium dodecyl sulfate (SDS) as surfactant, with a concentration equal to 10 times the surfactant critical micelle concentration (cmc). The initial liquid fraction is $\phi_{l} \simeq 5 \%$. Complementary tests were made using a more complex chemical formulation, providing a high interfacial viscoelasticity [32].

\section{B. Computations of correlation functions}

The raw data are a numbered succession of images of $N_{p}=13120$ pixels acquired at intervals $1 / 4 f=312.5 \mu \mathrm{s}$. Because the light intensity levels are low, we need to carefully remove the electronic noise of the sensor. We define the normalized intensity correlation function between two images $n$ and $m$ as:

$$
g_{I}^{n, m}=\frac{\left\langle I_{m} I_{n}\right\rangle-\left\langle I_{m}\right\rangle\left\langle I_{n}\right\rangle-a_{\text {elec }}-b_{\text {elec }} \delta_{n m}}{\left\langle I_{n}^{2}\right\rangle-\left\langle I_{n}\right\rangle^{2}-a_{\text {elec }}-b_{\text {elec }}}
$$

We noted $I_{n}$ the intensity vector of $N_{p}$ pixels of the image $n$, and $\langle\cdot\rangle$ designs here an average on all the pixels. We checked that the electronic noise $i_{n}$ is such that $\left\langle i_{n} i_{m}\right\rangle=a_{\text {elec }}+b_{\text {elec }} \delta_{n m}$. The values of $a_{\text {elec }}$ and $b_{\text {elec }}$ are determined by an independent measurement, and we checked that for a well aged foam at rest, we get $g_{I}^{n, m \gg n}=0$ and $g_{I}^{n, n+1}=1$.

For a cycle of oscillation $n$, we compute the quantities 
$g_{I}^{(i)}(n)$ as:

$$
\begin{aligned}
g_{I}^{(0)}(n) & =\frac{1}{4} \sum_{p=0}^{p=3} g_{I}^{4 n+p, 4 n+4+p} \\
g_{I}^{(1)}(n) & =\frac{1}{2}\left(g_{I}^{4 n, 4 n+2}+g_{I}^{4 n+2,4 n+4}\right) \\
g_{I}^{(2)}(n) & =\frac{1}{2}\left(g_{I}^{4 n+1,4 n+3}+g_{I}^{4 n+3,4 n+5}\right) \\
g_{I}^{(3)}(n) & =\frac{1}{2}\left(g_{I}^{4 n, 4 n+1}+g_{I}^{4 n+1,4 n+4}\right) \\
g_{I}^{(4)}(n) & =\frac{1}{2}\left(g_{I}^{4 n+1,4 n+2}+g_{I}^{4 n+2,4 n+5}\right) .
\end{aligned}
$$

If we suppose a scattering medium where the dynamics are only due to the acoustic wave (i.e. no internal dynamics), we have for $i \neq 0$ :

$$
g^{(i)}=\sqrt{g_{I}^{(i)}(4 n)}
$$

where the $g^{(i)}$ are the quantities defined in (10). The interest of computing $g_{I}^{(0)}(n)$ and of the somewhat tricky definitions of $g_{I}^{(i \neq 0)}(n)$ is to allow some corrections of the scattering medium dynamic. This will be explained in Sec. IV A.

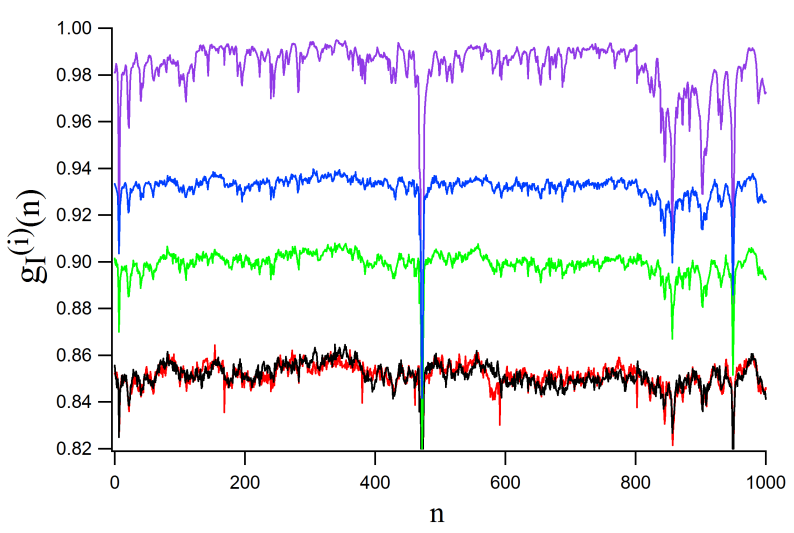

FIG. 3. Correlations functions of the scattered intensities $g_{I}^{(i)}(n)$ as a function the number $n$ of the oscillation cycle. Colors are $i=0$ : purple, 1: black, 2: red, 3: green and 4: blue.

Fig. 3 shows the evolution of the function $g_{I}^{(i)}(n)$ as a function of the cycle of oscillation $n$. In this experiment the value of the acoustic excitation is kept constant, with a fixed phase. $g_{I}^{(0)}$ is close to 1 , the fluctuations being due to the intrinsic dynamics of the foam (coarsening events). The values of $g_{I}^{(i \neq 0)}$ fluctuate around their mean values, and the values depend on the partial correlations functions number $i$.

In the following, we will average $g^{(i)}$ on 250 cycles of oscillations, i.e. on sequences of acquisition $0.31 \mathrm{~s}$.

\section{Amplitude and phase measurements}

In this Section, we demonstrate that we can determine the imposed variation of amplitude and phase - at a given location - from our model of combinations of correlations functions.

The amplitude of the strain is obtained from (12a) as: $\gamma^{2}(\mathbf{r})=C \times\left[2-g^{(1)}-g^{(1)}\right]$. We perform experiments where the amplitude of the strain is varied. For this, we change the level of excitation of the loudspeaker. In our experiments, we remain always below $90 \mathrm{~dB}$ of acoustic level in air, hence the pressure amplitude in air is below $1 \mathrm{~Pa}$, and it is even lower in foam because only a fraction of acoustic energy is transmitted to the foam. Hence, we remain in the regime of linear acoustics. Indeed, since foam is mostly made of gas, the order of magnitude of its bulk modulus is given by the atmospheric pressure, $10^{5} \mathrm{~Pa}$, and deviations from linear acoustics are expected only when the pressure amplitude of the acoustic wave becomes a significant fraction of the bulk modulus.

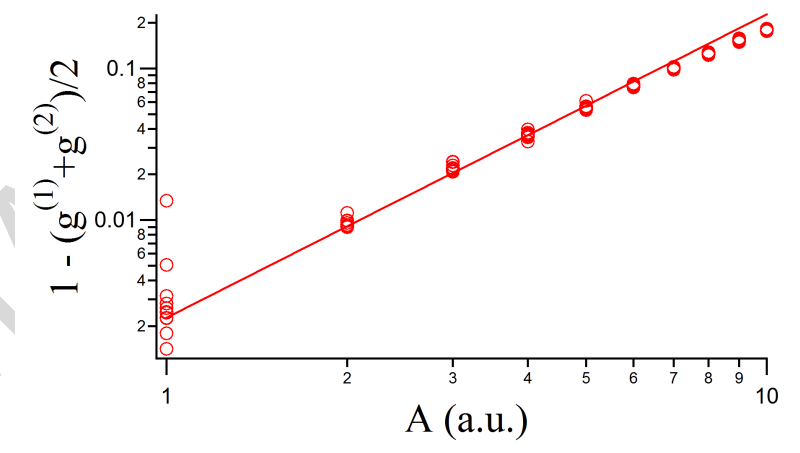

FIG. 4. Symbols: $1-\left(g^{(1)}+g^{(2)}\right) / 2$ as a function of the amplitude of excitation of the acoustic wave. The straight line is of slope 2 .

Fig. 4 shows the evolution of $1-\left(g^{(1)}+g^{(2)}\right) / 2$ as the function of the excitation amplitude. Every symbol correspond to one measurement of duration $0.31 \mathrm{~s}$. We see that the decorrelation increases with the amplitude of the acoustic excitation. The straight line is line of slope 2 , showing that $1-\left(g^{(1)}+g^{(2)}\right) / 2$ varies as $\gamma^{2}$ at least at small amplitudes. This is in agreement with (4). It should be remained that (4) has been obtained in the limit of small phase shifts, and deviations to the $\gamma^{2}$ are expected at large strain amplitudes.

The phase of the strain is obtained from (12b). In order to test this relation, we proceed as follow: we fix the amplitude of the acoustic wave, and we begin to acquire images with a given phase shift $\psi_{0}^{(i m p)}$ (see Sec. III A). Fig. 5a shows the evolution of $g^{(i)}$ as a function of the imposed phase shift $\psi_{0}^{(i m p)}$. We see that the correlation functions evolves with the imposed phase shift. As it may be seen from (9) and (10), advancing the imposed phase of $\pi / 2$ has the same effect of the permutations of $g^{(1)}$ with $g^{(2)}$ and of $g^{(3)}$ with $g^{(4)}$, reflecting the $\omega t+\psi_{0}$ 

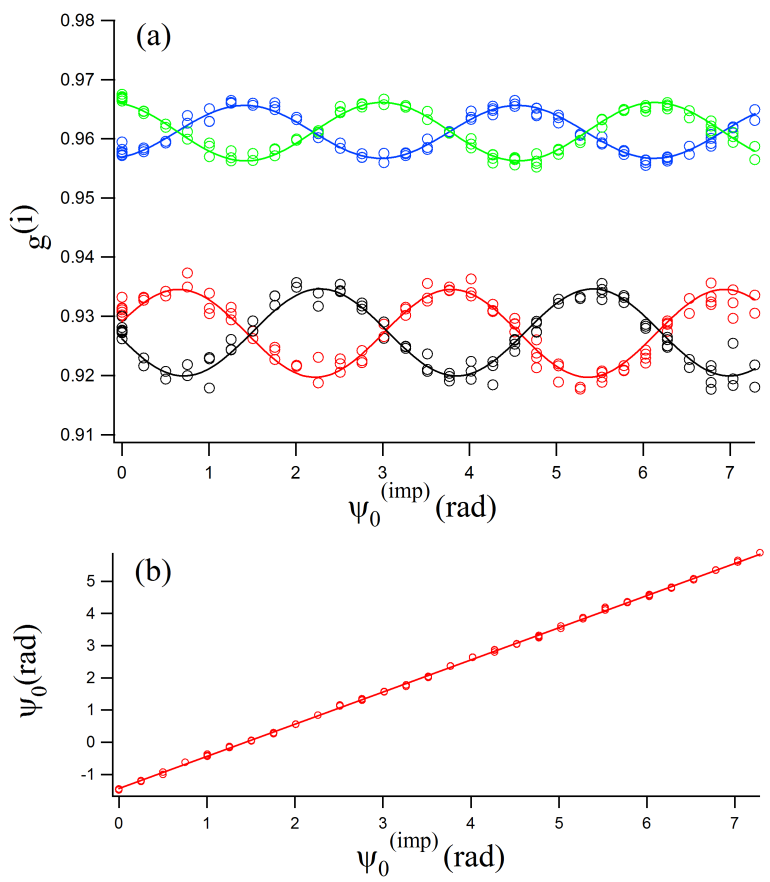

FIG. 5. (a) Variations of $g^{(i)}$ as a function of an imposed phase shift. $i=1$ : black, 2: red, 3: green and 4: blue. Symbols are results from measurements and continuous curves are sinusoids of period $\pi$ for eyes guidelines. (b) Computed phase shift as a function of the imposed one. Symbols are measurements and the line is the linear fit with a slope 1.

dependence of (9). The phase is obtained from (12b) as:

$$
\Psi_{0}=\frac{1}{2} \operatorname{arccot}\left[\left(\frac{g^{(1)}-g^{(2)}}{2-g^{(1)}-g^{(2)}}\right) /\left(\frac{g^{(3)}-g^{(4)}}{2-g^{(3)}-g^{(4)}}\right)\right]
$$

The unwrapped phase $\Psi_{0}$ calculated from the data of Fig. 5a is plotted on Fig. 5b. As it may be seen, the phase of the acoustic wave is measured with high precision. This shows that the phase of the strain may be extracted from DWS synchronized with the strain source with great accuracy.

\section{Application to the measurement of phase velocity in a foam}

Building on the previous experimental proofs of concept at a single position, we investigated how the phase evolve inside a foam as a function of the distance of propagation into our cylindrical cell. This is the relevant experiment in terms of foam acoustics, as it should provide information which can be compared to previous ones. In practice, the laser and the camera are therefore translated of a distance $\Delta x=2 \mathrm{~mm}$ between successive measurements. The ageing time of the foam is here $45 \mathrm{mn}$. This is a short enough time to avoid drainage and to prevent liquid fraction variations in the scanned part of the foam [33].

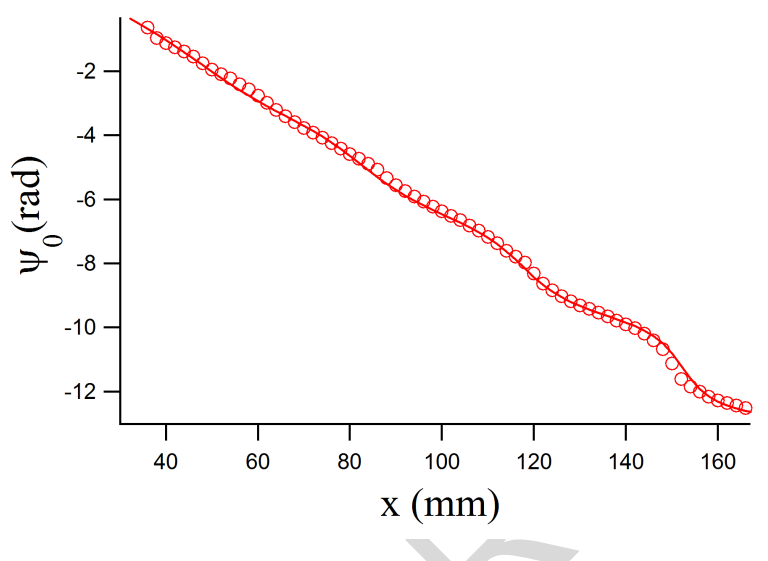

FIG. 6. Sound phase as a function of the distance of propagation: comparison between the measured phase (symbol) and the model of Eq. (17).

Fig. 6 shows the evolution of the phase as a function of the depth. The phase shift is roughly $4 \pi$, corresponding to two acoustic wavelengths. In this experiment, the bottom "wall" is the top surface of the drained liquid in the bottom of the glass cylinder. It is located at a the distance $H=202 \mathrm{~mm}$. We see that, far from the bottom of the foam, the phase evolves linearly with the distance; however, small oscillations of the phase occurs as the surface of the drained liquid is approached. Rather than only focusing on the linear regime away from the bottom, we can take into account all these features by including interferences between the propagating and reflected wave into the cylinder. Let $u_{i}(x)=u_{0} \exp \left[-\left(j k_{a}+\beta_{a}\right) x\right]$ be the incident acoustic wave, with $u_{0}$ the amplitude, $k_{a}$ the wave vector of the acoustic wave and $\beta_{a}$ the attenuation coefficient. The reflected wave is $u_{r}(x)=R u_{0} \exp \left(j k_{a}+\beta_{a}\right) x$ with $R$ the complex reflection coefficient. We set that, at the bottom, the drained liquid is a perfectly reflecting surface, which yields the boundary condition: $u_{r}(H)+u_{i}(H)=0$. This assumption comes from the fact that the acoustic impedance $Z=\rho c$ of a foam is orders of magnitude lower than that of the liquid, because both $\rho$ and $c$ are much lower in foam than liquid. It follows that $R=e^{-2\left(j k_{a}+\beta_{a}\right) H}$, and that the argument of $u=u_{r}+u_{i}$ is:

$\arg u=-j k_{a} x-\arctan \frac{e^{-2 \beta_{a}(H-x)} \sin 2 k_{a}(H-x)}{1+e^{-2 \beta_{a}(H-z)} \cos 2 k_{a}(H-x)}$.

The plain line of Fig. 6 shows the evolution of $\arg (u)$ as the function of the distance as predicted by (17). The free parameters are $k_{a}, \beta_{a}$ and a constant phase which is added to $\arg (u)$. The shown adjustment is obtained with $k_{a}=0.09 \mathrm{~mm}^{-1}$ and $\beta_{a}=0.009 \mathrm{~mm}^{-1}$. The value of the velocity is thus $v=\omega / k_{a}=56 \mathrm{~m} / \mathrm{s}$. These values fully agree with the previous studies. For the velocity, it is expected to have a value following the Wood's law, valid 
for these bubble sizes and frequencies [18, 19]. Indeed, the Wood's law predicts a velocity of $55 \mathrm{~m} / \mathrm{s}$ for $\phi_{l}=0.05$. Although we have not monitored the variation of liquid fraction, the fact that this value is so close to the experimental value also suggests that drainage has not modified much the profile of liquid fraction over the height of the foam probed in Fig. 6. Also, Pierre et al. [19] have shown by acoustic measurements in an impedance tube that at $800 \mathrm{~Hz}$, the attenuation coefficient equals $8 \pm 1 \mathrm{~m}^{-1}$ for SDS foams, a value insensitive (at this frequency) to the liquid fraction, which is in excellent agreement with our measurement.

\section{REMARKS ON THE METHODS AND ON THE EXPERIMENTAL TESTS}

Following the experimental tests demonstrating the feasibility of measuring the amplitude and the phase of a acoustic wave propagating into a foam, we want to point out a few other remarks on this synchronized DWS method.

\section{A. Corrections of internal dynamics}

We want here to give some information about the sensitivity of the determination of the correlation functions. The first point to address is the effect of the internal dynamics of the scattering medium on the correlation functions. Fig. 3 shows the evolutions of the correlation functions $g_{I}^{(i)}$ with time. The fluctuations are due to the internal dynamics of the system. Indeed, it is well known that reorganizations occurs in foam which create variations on path lengths $[10,11]$. When those fluctuations of correlations are small compared to the decorrelations due to the acoustic wave, their effects may be safely neglected. However, in the case of very small strains, their effects must be taken into account. A simple way consists in considering that the internal and the acoustic waves dynamics are uncorrelated. Defining $\Delta \phi_{\text {int }}(t, t+\tau)$ the variation of phase due to the internal dynamic, (4) becomes:

$g_{E}(t, t+\tau) \simeq 1-A\left[\left\langle\Delta \phi^{2}(t, t+\tau)\right\rangle+\left\langle\Delta \phi_{i n t}^{2}(t, t+\tau)\right\rangle\right]$

with $\Delta \phi(t, t+\tau)$ the phase shift due to the acoustic wave. Since $\Delta \phi(t, t+T)=0$, we have $g_{E}(t, t+T) \simeq$ $1-A\left\langle\Delta \phi_{\text {int }}^{2}(t, t+T)\right\rangle$. If the internal dynamic is due to many independent rearrangements which occurs at a constant rate between $t$ and $t+T$, we expect that $\left\langle\Delta \phi_{\text {int }}^{2}(t, t+\tau)\right\rangle=(\tau / T)\left\langle\Delta \phi_{\text {int }}^{2}(t, t+T)\right\rangle$, and then:

$$
\begin{aligned}
& 1-A\left\langle\Delta \phi^{2}(t, t+\tau)\right\rangle \simeq \\
&\left.g_{E}(t, t+\tau)+\frac{\tau}{T}\left[1-g_{E}(t, t+T)\right)\right] .
\end{aligned}
$$

Since all the correlation functions $g_{I}^{(i)}$ with $i \neq 0$ are measured on a the same mean delay $\tau=T / 2$, we may re- move the internal dynamics by computing a "corrected" value:

$$
g_{I ; \text { corr }}^{(i \neq 0)}=\sqrt{g_{I}^{(i \neq 0)}(4 n)}+\frac{1}{2}\left[1-\sqrt{g_{I}^{(0)}(4 n)}\right] .
$$
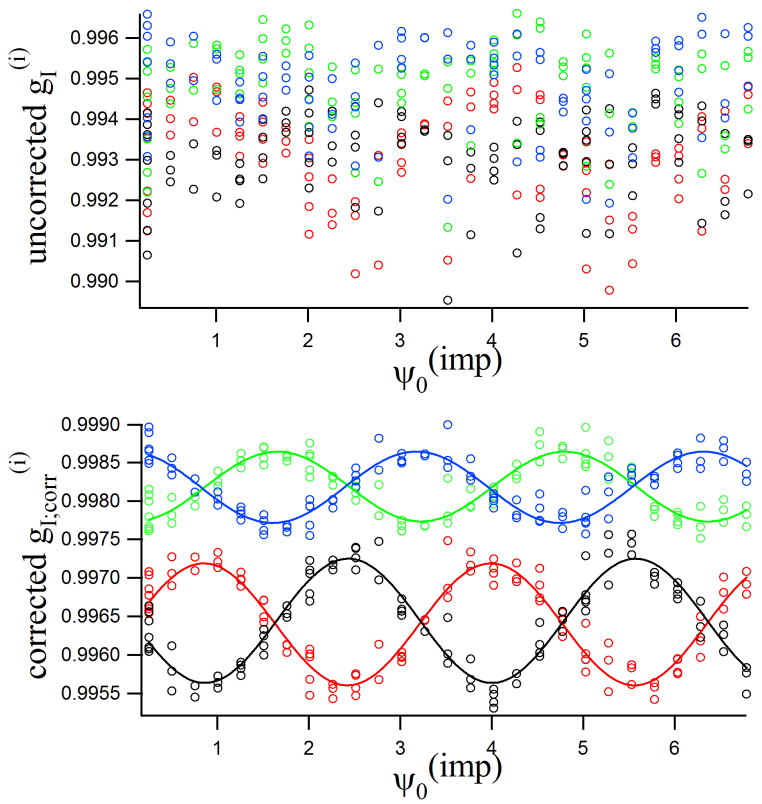

FIG. 7. Symbols: uncorrected (top) and corrected (bottom) values of $g_{I}^{(i)}$ as a function of an imposed phase shift. Curves are sinusoidal guidelines. Color codes are the same as for Fig. 3

The effect of such a correction is striking on a small amplitude measurement. Fig. 7 shows a measurement where the phase is controlled and the amplitude of the acoustic wave is very small. If the internal dynamics is not corrected, one can hardly detect the evolutions of $g^{(i)}$ with the imposed phase. However as far as the internal dynamics is corrected using (20), the variations are clearly visible. This shows that acoustic propagation may be probed even if the internal dynamic dominates the variations of path lengths.

\section{B. Sensitivity and comparison with the stroboscopic method}

We want here to quantify the uncertainties on the determination of the amplitude and of the phase. We focus on the noise on measurements during 250 oscillations cycles. Due to the multispeckle scheme used here, the measurement of correlation functions are averaged on many coherence areas. We record images of 13120 pixels, and the coherence area of $\approx 4$ pixels. We then have $\approx 3000$ coherence areas on every image. Because the scattering medium used here has a internal dynamics, the speckle pattern evolves slowly. The typical timescale of this evo- 
lution may be measured in a experiment where no oscillations take place, and we found an evolution time $\approx 0.1 \mathrm{~s}$, which is $1 / 3$ of the experiment duration. With both the time and the multispeckle averages, one phase and amplitude measurement is averaged on $\approx 3 \times 3000$ realizations.

The noise for the phase determination is estimated as the difference between the measured and the imposed phase in a experiment where the phase is imposed. We found a typical noise of $0.02 \mathrm{rad}$, which does not seem to depend strongly on the amplitude of oscillation. The standard deviations on the values of $1-\left(g^{(1)}+g^{(2)}\right) / 2$ are typically of order $10^{-3}$ when signals are uncorrected from internal dynamics and of $2 \times 10^{-4}$ when the signals are corrected. We may then expect to measure $1-g_{E}$ on typically 3 orders of magnitude.

An alternative method to characterize mechanical wave in light diffusing medium has been proposed recently by Wintzenrieth et al [23]. When a scattering medium is submitted to a strain modulation, the contrast of the time-averaged speckle pattern is lowered compared to contrast of the same static medium. This has been used to detect ultrasonic waves in optical tomography measurements [34]. By synchronizing the beginning of the light acquisition with the acoustic wave, and by measuring the visibility of the speckle pattern, the phase of the strain modulation may be obtained [23]. A quantitative comparison between this method and the method reported in this study is not straightforward. Such a comparison requires quantitative models for the different sources of noises, for instance noises due to internal dynamics of the scattering medium, or noise due to sensors, and this analysis is far from being trivial. Nevertheless, we may notice that the visibility methods correspond to measurements of correlation functions integrated over time. If $g_{E}(t)$ is the electric field correlation function the visibility is $V(T)=\frac{1}{T} \int_{0}^{T} 2\left(1-\frac{t}{\tau}\right)\left|g_{E}(t)\right|^{2} d t$ [9]. It is then easy to show that $g_{E}(t)$ is related to the second time derivative of the visibility: $\left|g_{E}(t)\right|^{2}=\frac{1}{2} \frac{d^{2}\left[V(t) t^{2}\right]}{d t^{2}}$. Generally speaking, a time derivative is a source of noise. Hence, we may expect that synchronized acquisitions, which avoid such derivatives, allow to obtain correlation functions with a better signal-to-noise ratio. This is in agreement with the fact that we are able to measure $1-g_{E}$ on typically 3 orders of magnitude, while Wintzenrieth et al. [23] obtains a dynamical range for $1-g_{E}$ of one order of magnitude. This is also confirmed by numerical simulations where we compared correlations functions obtain directly by correlation of signal, and by correlation functions obtained by time-derivative of visibility. However, only an experimental comparison of the two methods on different experimental systems may quantitatively confirm these differences.

\section{Structure of the sheared layer}

The method explained in this paper permits to measure the amplitude and the phase of a sheared zone. We discuss in this subsection the origin and the structure of the shear created by an acoustic wave near a solid surface which is perpendicular to the propagation direction of the acoustic wave. In a previous paper [22], we have considered the experimental situation of an acoustic wave propagating into a foam. The wave propagates parallel to the wall. Because of the non-slip boundary condition at the boundary, the acoustic displacement cancels at the wall. The variation of the displacement with the distance from the wall occurs in a viscoelastic boundary layer, which depends on the rheology of the material. We treat the foam as a continuous visco-elastic fluid, with $\rho$ the density and $\underline{G}=G_{0} \exp j \Delta$ the visco-elastic modulus of the material. Let $u(z, t)=\operatorname{Re}\left[\underline{u}(z) e^{i \omega t}\right]$ be the acoustic displacement, and $u_{0}=\lim _{z \rightarrow \infty} \underline{u}(z)$ the displacement far from the wall. The force balance writes:

$$
\rho \frac{\partial^{2} \underline{U}(z, t)}{\partial t^{2}}=\underline{G} \frac{\partial^{2} \underline{U}(z, t)}{\partial z^{2}},
$$

with $\underline{U}(z, t)=\underline{u}(z, t)-u_{0}$. The solution of $(21)$ is:

$$
\underline{U}(z, t)=U_{0} \exp (j \omega t) \exp (\underline{\kappa} z),
$$

with $U_{0}+u_{0}$ the amplitude of displacement at $z=0$ and $\underline{\kappa}=\kappa_{0} \exp j \varphi$. We have $\kappa_{0}=\omega \sqrt{\rho / G_{0}}$ and $\varphi=(\pi-\Delta) / 2$. The strain is $\underline{\gamma}=\partial \underline{U}(z, t) / \partial z=$ $U_{0} \underline{\kappa} \exp (j \omega t) \exp (\underline{\kappa} z)$, and we obtain:

$$
\begin{aligned}
& |\underline{\gamma}(z)|=\kappa_{0} \exp \left[-\kappa_{0} \cos (\varphi) z\right] \\
& \Psi_{0}(z)=\varphi+\kappa_{0} \sin (\varphi) z
\end{aligned}
$$

In this model, the amplitude and the phase of the strain depend on $z$. As explained in [22], it is this sheared layer that is probed with light scattering. In Sec. II, we made the hypothesis that the deformation is homogeneous into the volume probed by the light. Using this hypothesis, every term of the sum $\sum_{j=1}^{s / l^{*}}\left\langle\Delta \phi_{j}^{2}\right\rangle$ of phase shift used in (3) is the same: $\left\langle\Delta \phi^{2}\right\rangle$. The deformation being in fact heterogenous, we must consider average values of $\left\langle\Delta \phi^{2}\right\rangle$ on volumes $\mathcal{V}_{s}$ probed by paths of length $s$ : $\sum_{j=1}^{s / l^{*}}\left\langle\Delta \phi_{j}^{2}\right\rangle=\left(s / l^{*}\right)\left\langle\Delta \phi^{2}\right\rangle_{\mathcal{V}_{s}}$. Because long paths probe larger volumes than short paths, the volume $\mathcal{V}_{s}$ depends on $s$.

The fact that the phase of the strain is heterogeneous has an interesting consequence. Indeed, let us consider a segment of the random walk located at a distance $z$ from the wall. The variance of the phase shift (8) depends on $z$ :

$$
\left\langle\Delta \phi^{2}(z)\right\rangle \propto \gamma^{2}(z) \sin ^{2}(\omega \tau / 2) \sin ^{2}\left[\omega t+\omega \tau / 2+\Psi_{0}(z) \psi^{4} 4\right)
$$

Since $\Psi_{0}$ depends on $z,\left\langle\Delta \phi^{2}(z)\right\rangle$ cannot be canceled simultaneously at every $z$. It follows that the correlation function $g^{(i)}$ with $i \neq 0$ cannot be equal to one. This is what is observed on the Fig. 5a: the maximum values of the correlation functions are not 1 . Complementary experiments with foam of high interfacial viscoelasticity [32] exhibit higher values of correlation recovery. This property may be used as a useful non-destructive method to 
estimate the value of $\varphi$ and will be discussed in a forthcoming paper.

Finally, we can justify the assumption on the displacement field made to derive (7) from (6). At the frequency of $800 \mathrm{~Hz}$, the thickness $\xi=1 / \kappa_{0} \cos (\varphi)$ of the sheared layer is about $2 \mathrm{~mm}[22]$, whereas the acoustic wavelength in the foam is $\lambda_{a}=2 \pi / k_{a}=6 \mathrm{~cm}$ (see Sec. III D). Hence, $\xi / \lambda_{a} \ll 1$, and to a good approximation, the displacement field in the sheared layer writes : $\mathbf{A}(\mathbf{r}, t)=[\alpha(z) \cos (\omega t)+\beta(z) \sin (\omega t)] \mathbf{e}_{x}$, with $\mathbf{e}_{x}$ the unit vector in the direction of propagation. Hence, $\nabla \alpha$ and $\boldsymbol{\nabla} \beta$ are both along $\mathbf{e}_{z}$.

\section{CONCLUSION}

We have investigated the situation of a turbid media undergoing a periodical shear, and wondered if and how the amplitude and the phase of this deformation could be locally measured by light scattering. We have proposed a new approach - named here "synchronized DWS" which requires to acquire four different correlations functions. Then by combining these correlations, the amplitude and the phase can be derived, at any locations. This approach has been successfully tested by experiments on foams, where a sinusoidal shear is obtained as an acoustic wave is propagating. In a first set of tests, we have found an excellent agreement between the imposed forcing and the measurements. These first results fully validated the principles and our formalism. Further experiments allowed us to scan a foam as a function of the distance of propagation of the sound. It turns out that we have been able to monitor the phase of the deformation along a few wavelengths. The full analysis - including in- terference effects at the bottom of the vessel - allowed us to quantify the phase velocity and the sound attenuation. The obtained values are in excellent agreement with previous measurements, and definitively confirms that this approach based on the measurement by DWS of the acoustic deformation is relevant. Moreover, we have shown that possible internal dynamics and heterogeneity in space do not prevent these types of measurements, as they can also be taken into account.

In the future, these results can be pursued along different routes. For instance, this method may be used to get information about the shear layer in a direction perpendicular to the displacement. We saw in Sec. IV C that the structure of this sheared layer depends on the rheological properties of the material. Correlation data should then permit to obtain informations about high frequency rheological properties with $\lambda_{a} \lesssim l^{*}$. A deeper understanding will be required to clarify the links between the correlation data and the viscoelasticity of the material. As well, measurements can be done not only in the direction of propagation, but also in the orthogonal one. It is thus possible to create a 2D mapping of the amplitude and of the phase; this might be especially relevant in the cases where sound could be diffracted by obstacles or slits.

\section{ACKNOWLEDGMENTS}

We acknowledge funding support from Agence Nationale de la Recherche in the frame of the SAMOUSSE project (ANR-11-BS09-001, coordinated by Florence Elias). We thank all the participants of this project for fruitful discussions. We also thank the GdR "Mousses et Émulsions" for providing another network for discussions.
[1] Pine, D.J., Weitz, D.A., Zhu, J.X., and Herbolzheimer, E. Diffusing-wave spectroscopy: dynamic light scattering in the multiple scattering limit. J. Phys. France, 51(18):2101-2127, 1990.

[2] In W. Brown, editor, Dynamic Light Scattering: The Method and Some Applications. Oxford University Press, 1993.

[3] S. Kirsch, V. Frenz, W. Schartl, E. Bartsch, and H. Sillescu. J. Chem. Phys., 104:1758, 1996.

[4] L. Cipelletti and D. Weitz. Rev. Sci. Instrum., 70:3214, 1999.

[5] V. Viasnoff, F. Lequeux, and D.J. Pine. Rev. Sci. Instrum., 73:2336, 2002.

[6] L. Cipelletti, H. Bissig, V. Trappe, P. Ballesta, and S. Mazoyer. J. Phys.: Condens. Matter, 15:S257, 2003.

[7] P.K. Dixon and D.J. Durian. Phys. Rev. Lett., 90:184302, 2003.

[8] K.N. Pham, S.U. Egelhaaf, A. Moussaid, and P.N; Pusey. Rev. Sci. Instrum., 75:2419, 2004.

[9] R. Bandyopadhyay, A. S. Gittings, S. S. Suh, P. K. Dixon, and D. J. Durian. Speckle-visibility spectroscopy: A tool to study time-varying dynamics. Review of Scientific In- struments, 76(9):-, 2005.

[10] D. J. Durian, D. A. Weitz, and D. J. Pine. Scaling behavior in shaving cream. Phys. Rev. A, 44:R7902-R7905, Dec 1991.

[11] D. J. Durian, D. A. Weitz, and D. J. Pine. Multiple light-scattering probes of foam structure and dynamics. Science, 252(5006):686-688, 1991.

[12] Reinhard Hohler, Sylvie Cohen-Addad, and Douglas J. Durian. Multiple light scattering as a probe of foams and emulsions. current opinion in colloid and interface science, 2014.

[13] R. Hohler and S. Cohen-Addad. J. Phys. Condens. Matter, 17:R1041, 2005.

[14] R. K. Prud'homme and S.A. Khan. Foams: Theory, Measurements, and Applications. OMarcel Dekker Inc., New York, 1997.

[15] I. Cantat, S. Cohen-Addad, F. Elias, F. Graner, R. Hohler, O. Pitois, F. Rouyer, and A. Saint-Jalmes. Foams - Structure and Dynamics. Oxford University Press, 1 edition, 2013.

[16] K. B. Kann. Sound waves in foams. Colloids Surf. A, 263:315-319, 2005. 
[17] I. Ben Salem, R.-M. Guillermic, C. Sample, V. Leroy, A. Saint-Jalmes, and B. Dollet. Propagation of ultrasound in aqueous foams: bubble size dependence and resonance effects. Soft Matter, 9:1194-1202, 2013.

[18] J. Pierre, F. Elias, and V. Leroy. A technique for measuring velocity and attenuation of ultrasound in liquid foams. Ultrasonics, 53:622-629, 2013.

[19] J. Pierre, R.-M. Guillermic, F. Elias, W. Drenckhan, and V. Leroy. Acoustic characterization of liquid foams with an impedance tube. Eur. Phys. J. E, 36:113, 2013.

[20] J. Pierre, B. Dollet, and V. Leroy. Resonant acoustic propagation and negative density in liquid foams. Physical Review Letters, 112:148307, 2014.

[21] J. Pierre, B. Giraudet, P. Chasle, B. Dollet, and A. SaintJalmes. Sound propagation in liquid foams: Unraveling the balance between physical and chemical parameters. Phys. Rev. E, 91:042311, 2015.

[22] M. Erpelding, R. M. Guillermic, B. Dollet, A. SaintJalmes, and J. Crassous. Investigating acoustic-induced deformations in a foam using multiple light scattering. Phys. Rev. E, 82:021409, 2010.

[23] Frédéric Wintzenrieth, Sylvie Cohen-Addad, Marie Le Merrer, and Reinhard Höhler. Laser-speckle-visibility acoustic spectroscopy in soft turbid media. Phys. Rev. E, 89:012308, Jan 2014.

[24] S. Lévêque, A. C. Boccara, M. Lebec, and H. SaintJalmes. Ultrasonic tagging of photon paths in scattering media:?parallel speckle modulation processing. Opt. Lett., 24(3):181-183, Feb 1999.

[25] Gang Yao and Lihong V. Wang. Theoretical and exper- imental studies of ultrasound-modulated optical tomography in biological tissue. Appl. Opt., 39(4):659-664, Feb 2000.

[26] Linda Djaoui and Jerome Crassous. Probing creep motion in granular materials with light scattering. Granular Matter, 7(4):185-190, 2005.

[27] D Bicout, E Akkermans, and R Maynard. Dynamic correlations for multiple light-scattering in laminar-flow. JOURNAL DE PHYSIQUE I, 1(4):471-491, APR 1991.

[28] D Bicout and R Maynard. Diffusing Wave Spectroscopy in inhomogeneous flows. PHYSICA A, 199(3-4):387-411, NOV 11993.

[29] W Leutz and G Maret. Ultrasonic Modulation if multiply scattered-light. PHYSICA B, 204(1-4):14-19, JAN 1995.

[30] A. Saint-Jalmes, M.U. Vera, and D. J. Durian. Eur. Phys. J. $B, 12: 67,1999$.

[31] A. Saint-Jalmes. Soft Matter, 2:836, 2006.

[32] K. Golemanov, N. D. Denkov, S. Tcholakova, M. Vethamuthu, and A. Lips. Surfactant mixtures for control of bubble surface mobility in foam studies. Langmuir, 24(18):9956-9961, 2008. PMID: 18698860.

[33] Arnaud Saint-Jalmes and Dominique Langevin. Time evolution of aqueous foams: drainage and coarsening. Journal of Physics: Condensed Matter, 14(40):9397, 2002.

[34] Roger Zemp, Sava Sakadžić, and Lihong V. Wang. Stochastic explanation of speckle contrast detection in ultrasound-modulated optical tomography. Phys. Rev. E, 73:061920, Jun 2006. 